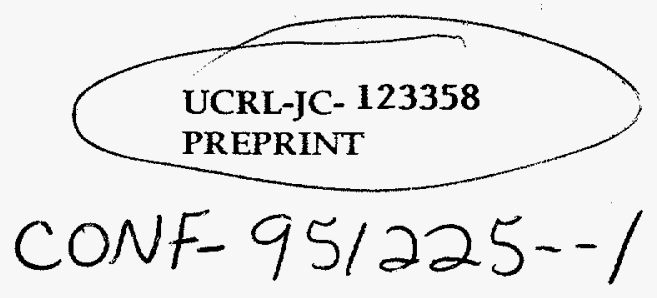

\title{
Proper Motion with HST: Searching for High-Velocity Stars in the Core of the Globular Cluster 47 Tucanae
}

Georges Meylan, Dante Minniti, Carlton Pryor, Christopher G. Tinney, E. Sterl Phinney, Bruce Sams
RECEIVED

MAR $28 \% 996$

OSTI

This document was prepared for submittal to Science with the Hubble Space Telescope - II

Space Telescope Science Institute, 1996 Conference,

Paris, France,

December 4-8, 1995

February 13, 1996

This is a preprint of a paper intended for publication in a journal or proceedings. Since changes may be made before publication, this preprint is made available with the understanding that it will not be cited or reproduced without the permission of the author.

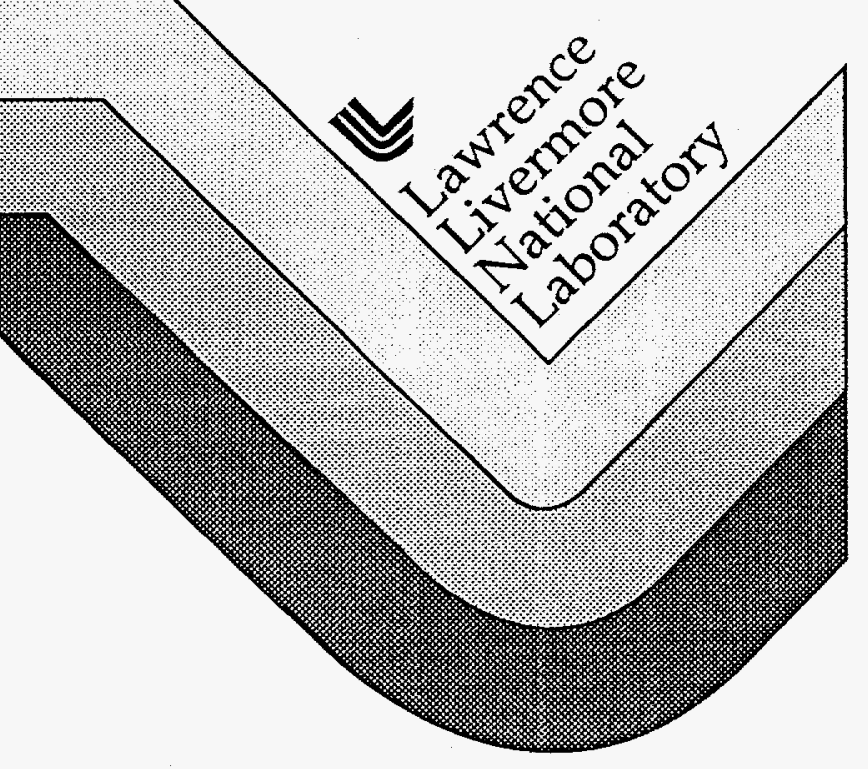




\section{DISCLAIMER}

This document was prepared as an account of work sponsored by an agency of the United States Government. Neither the United States Government nor the University of California nor any of their employees, makes any warranty, express or implied, or assumes any legal liability or responsibility for the accuracy, completeness, or usefulness of any information, apparatus, product, or process disclosed, or represents that its use would not infringe privately owned rights. Reference herein to any specific commercial products, process, or service by trade name, trademark, manufacturer, or otherwise, does not necessarily constitute or imply its endorsement, recommendation, or favoring by the United States Government or the University of California. The views and opinions of authors expressed herein do not necessarily state or reflect those of the United States Government or the University of California, and shall not be used for advertising or product endorsement purposes. 


\section{DISCLAIMIER}

Portions of this document may be illegible in electronic image products. Images are produced from the best available original document. 
Science with the Hubble Spacc Telescope - II

Space Telescope Science Instifute, 1996

P. Benvenuti, F. D. Macchetto, \& E. J. Schreier, eds.

\section{Filial version}

So not circulate.

Proper Motions with HST: Searching for High-Velocity Stars in the Core of the Globular Cluster 47 Tucanae

Georges Meylan

European Southom Obsermatory, Garching b. München. Gormumy

D) ante Minniti

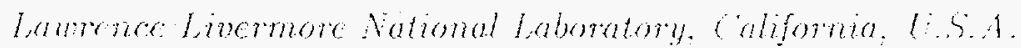

Carloum Pryor

Rutgers University, Dept of Physics and Astronomy. Ner Jorsy. T.S.A.

Christopher G. Tinney

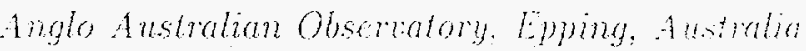

E. Sten Phinney

Theoretical Astrophysics. Caltech, Pasadena. L.S.4.

Bruce Sams

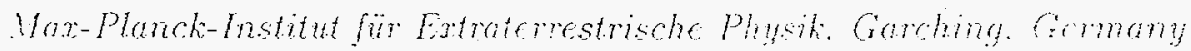

Abstract. Binary stars play an essential role during the late phases of the dymanical evolution of a globalar cluster. They transfer energy to passing stars and so can strongly influence the cluster evolution, enough to delay, halt. and even reverse core collapse. Hard binaries are known to exist in cluster cores, e.g.. in the form of milhisecond pulsars (about half of the 11 millisecond pulsars observed in 17 Tucallate are such hard binaries). The presence of hard binaries may also be revealed by searching for the by-products of close encounters: high-velocity stars, such as those discovered in the core of 47 Tuc by Mevlan et al. (1991) and Gebhardt et al. (1995). These studies represent the limit of the radial velocity data which can be obtancd from the gronnd. If more progress is to be made, it must be come through obtaning proper motions - a task for which only HST is sutable.

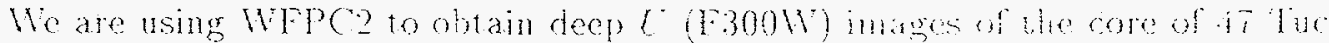
at thee diferent epochs over wo years, wh which we will measne differential proper motions to a l-o limit of 0.23 nas/yr. This - rather conservative estimate corresponds to a $5-\sigma$ detection of all stars with tangential velocities greater than $22 \mathrm{~km} \mathrm{~s}^{-1}$. By using the F300W filter we can measure stars over the whole colormagnitude diagram, from the red-giant branch to well down the main sequence. Such a complete census will provide unique constraints as a function of the stellar mass on relaxation processes, collision and ejection rates, and the velocity distribution.

Here we report on the first-epoch (Cycle 5) observations of this project. Although no proper motions are available yet, some preliminary by-product results are presented. These include luminosity functions and color-magnitude diagrams for the core of 47 Tuc and the light curves of variable blue straggler stars and of a candidate $X$-ray source. 


\section{Introduction}

\subsection{Binary Stars and Globular Cluster Dynamical Evolution}

One of the most intriguing features of globular cluster dynamical evolution is its instability. Whether viewed as arising from two-body relaxation (Hénon 1961), the mass-segregation instability (Spitzer 1969), or the gravothermal instability (Antonov 1962, Lynden-Bell \& Wood 1968), the evolution of the core of a globular cluster is expected to reach a state of very high density in a finite time: a phenomenon called "core collapse." The high densities resulting from core collapse increase both encounter and collision rates and can actually modify the stellar population in the cluster center, producing such exotic objects as binary $X$-ray sources, blue stragglers (BSs), and milli-second pulsars (see Meylan 1994 for a review) and possibly even affecting such traditional cluster properties as the shape of the horizontal branch (Djorgovski \& Piotto 1993). The stellar population can in turn influence the dynamical evolution.

It is now well established that binary stars control the late stages of core coliapse (see Hut et al. 1992 for a review). Gravitational encounters between hard binaries and passing stars leave the binary more tightly bound, with the released energy "heating" the binary"s envirnment. Binaries are concentrated in the core due to mass segregation and they can delay, hall, and even reverse the collapse. Primordial binaries provide a finite "fossil fuel" because the binaries are eventually destroyed by being driven to coalescence, by being distupted by an encounter with other binaries, or by being ejected from the cluster by the recoil from a strong interaction. At very high core densities, new binaries can be formed by encounters between three single stars (Heggie 1975) or by "tidal-capture", in which two stars approach so closely that they dissipate enough of their relative kinetic energy through tidal oscillations to remain bound (Fabian, Pringle, \& Rees 1975).

Enfortunately, the binary population in dense cluster cores is difficuli to study observationally: Because of crowding, radial velocity searches are confined to the few bright giants (e.g., Gebhardt et al. 1995). The broadening of the main sequence by binaries is only sensitive to systems with two luminous stellar components. Numerical simulations predict that many binaries in dense cores will contain at least one heavy, dark, white dwarf or neutron star. The numbers and kinds of exotic stellar objects in the core are a useful probe of the binary population because they are produced by stellar collisions that occur during gravitational encounters involving binaries. But these exotic objects are rare, making small number statistics a problem. Another possible probe of the binary population is suggested by the discovery of two high-velocity stars in the core of the globular cluster 47 Tuc (Meylan (et al. 1991). These stars very likely gained their high velocities from encounters with hard binarite.

\subsection{Tucanae}

One of the closest $\left(D \simeq 4.6 \mathrm{kpc} ;(m-M)_{Y}=13.3\right)$ and mos massive $\left(\mathcal{M} \simeq 10^{\circ} M_{0}\right)$ globular chsters in our Galaxy is 47 tuc. It has been intensively studied, both photometrically and hinematically: for decades. This cluster is relatively metal-rich $([F c / H] \simeq-0.7)$, has an age of $\simeq 13 \mathrm{Gyr}$ (Hesser et al. 1987), has low reddening $(E(B-V) \simeq 0.04)$, and has only small galactic contamination. The high concentration $(c \simeq 2.1)$ of 47 Tuc suggests that. it is dynamically evolved and, although well fitted by multi-mass anisotropic King-Michie models, that it is evolving through a sequence of quasi-equilibrium states towards its ultimate fate of core collapse (Meylan 1989). There is an increasing amount of direct and indirect observational evidence for stellar encounters and collisions in the core of 47 Tuc. The previously-known single X-ray source has been resolved by ROSAT into at least five components (Hasinger et al. 1994). Besides the high-velocity stars, other evidence for binarics in 47 Tuc comes from the eleven millisecond pulsars (Manchester et al. 1991, Robinson (2t. al. 1995) and the very high density of centrally clustered BSs observed by HST (Paresce 
et al. 1991). With one of the richest populations of collision products, 47 Tuc is clearly an ideal laboratory for confronting theoretical predictions with observations.

Radial velocities measured for 550 stars within two core radii of the center of 47 Tuc (Gebhardt et al. 1995) have increased the sample of high-velocity stars from 2 to 8 . With velocities more than $32 \mathrm{~km} \mathrm{~s}^{-1}$ from the cluster mean (about three times the core velocity dispersion of $\simeq 10 \mathrm{~km} \mathrm{~s}^{-1}$ ), these stars are moving at close to the cluster escape velocity. The original high frequency of rapidly moving stars ( 2 out of 50 ) stimulated theoretical studies of their expected numbers (Phinney \& Sigurdsson 1991, Sigurdsson \& Phinney 1995). While the larger sample yields a lower frequency, the studies have demonstrated that the number of high-velocity stars is a powerful tool for probing conditions in the core and testing the dynamical models. However, larger samples are needed to exploit this tool.

\subsection{Proper Motions with HST}

Consequently, we are using the Planetary Camera to obtain a complete census of highvelocity stars inside half a core radius of 47 Tuc, down to a limit of $U \simeq 22$. 'This census includes stars on the red giant branch (RGB), subgiant branch, red horizontal branch (RHB), upper and lower main sequence (down to $\sim \mathrm{K} 3 \mathrm{~V}$ ), and white dwarfs (see Figure 2 below). We do not need to take images with different filters, since good color information for the stars in the core of 47 Tuc is already available from previous HST programs (e.g. Guhathakurta et al. 1992).

Experience obtained with the techniques of CCD astrometry on the ground has shown thiat CCDs nake almost ideal devices for faint, relative astrometry. Astrometric positions with a precision of 5 mas/epoch are now being routinely measured from the ground (Monet et al. 1992; Timney 1993). In the absence of atmospheric seeing (the fundamental limitation for ground-based CCD astrometry), there is no reason why a suitably-designed astrometric program with VFPC2 cannot achieve precisions limited only by photon statistics. This means that measuring proper motions with $1-\sigma$ uncertanties of 0.2 mas/year should bo achievable. We have a conservative approach, aining first for the high-velocity stars, but. estimates show that we should be able to measure proper motions for most of the stars in our frames.

\subsection{Scientific Goals}

Given the strong limits imposed by seeing on the ground-based acquisition of radial velocities in the core of 47 Tuc, HST is the only telescope able to provide, in the very core of the cluster, a kinematic sample consisting of thousands of stars. The present census of proper motions will provide unique constraints on:

- relaxation processes (possible anisotropy of the velocity dispersion).

- collision and ejection rates (meaningful fraction of high-velocity stars from a large samplej.

- the form of the velocity distribution in the core as a lunction of the stellar mass, and

- mass segregation.

Natural by-products of this project already obtaned from plotometry of the PC.1. WF2, WF3, and WF4 frames are interesting per se and are discussed in Section 3 below.

\section{The Data}

\subsection{The Observations}

The fifteen WFPC2 frames constituting the first epoch observations for this project were obtained by HST on October 25, 1995 (Cycle 5), with the PC field centered on the core of 47 Tuc. Each exposure lasted 350 seconds and was taken through the F300W filter, which has a passband similar to $U$. The final combined image has total exposure time of 1.46 hour and reaches F300W $=25$ mag for $3-\sigma$ detections. 
We selected the F300W filter in order to reduce the range of brightness presented by the stars in the field, thus preventing the saturation of pixels by red giants and allowing more or less all stages of stellar evolution in the cluster to be seen. This filter allowrs the detection of main-sequence (MS) stars with a wide mass range simultaneously with RGB, RHB, AGB, BS, and white dwarf (WD) stars, without any of these stages being so bright compared to the others that multiple exposures with different observing times are required. For a similar number of stars detected, the dynamic range needed with the F $300 \mathrm{~W}$ filter is only about 6 magnitudes, compared with 10 magnitudes in the optical (c.g., between the RCBB and the WID sequence). The RHB and BS stars are brightest (see Figure 2). Sampling problems are also reduced in the UV compared with optical passbands. We prefer the wide I filter F300W to the Johnson $U$ filter F336W because of the higher throughput (a gain of about $0.5 \mathrm{mag}$ ) and a reduced red leak, both critical for the faintest objects. The red leak in the $\mathrm{P} 300 \mathrm{~W}$ filter is not a problem for our proposed science, since color information is a valable. The major disadvantage of the F $300 \mathrm{~W}$ filter is that it is less well ralibrated than other WFPC2 filters. Again, this does not affect our scientific results.

\subsection{The Photometry}

The phommery us obtained with the DAOPHOT II package within IRAF About 8,000 stars were identified in the PCl field and about 23,000 stars in the other three (WF2, WF3, WF4) fields, for a total of more than 30,000 stars. The results reported here were obtained using aperture photometry with a 2-pixel radius and the sky defined by an anmulus with 10. and 15-pixel radii. We followed the calibration procedure described by Holtzman et al. (1995, their Table 10). Typical photometric errors are $\sigma_{F 300 w}=0.01,0.04$, and 0.25 at $\mathrm{F} 300 \mathrm{~W}=16,20$, and 23 , respectively. Based on artificial star experiments, we estimate that the data are $100 \%$ complete down to $\mathrm{F} 300 \mathrm{~W}=19$ and $50 \%$ complete at $\mathrm{F} 300 \mathrm{~W}=22$.

\subsection{The Astrometry: Measuring Proper Motions with HST}

CCD astrometry from the ground has shown that three fundamental issues must be addressed in designing the observing program for high-precision relative astrometry. Once a.l three issues are solved, obtaining positions accurate to better than $1 \%$ of a pixd can easily be achieved. provided that the required photon counting limits $(\approx 10,000$ photons $)$ can be reached.

- The first requirement is that the astrometry must be carried out in a differential mode. Traditional photographic astrometry has always been limited by the need for a detailed knowledge of the field distortions (if the field is not flat). If it cannot be guaranteed a mim where the astrometric stars will be placed in that field, it is essential to be able kn flatten the fied io tigh precision. On the ground, CCDs obviate this problem by allowing one to alwats place the target objects back on the same place on the CCD to within a fow pixels, at which point the field distortions cancel out and high precision can be reached. This strategy is adopted for our observations: all of our frames are made with an identical roll angle and by placing the stars at the same position on the PC1.

- The second requirement is that the PSF must be well-sampled by the CCD. It has been realized in recent years that CCDs do not have uniform sensitivity across a pixel. In the worst case, the sensitivity can vary by as much as $5 \%$ across a single pixel. This obviously has serious implications for a program which aims to measure positions to an accuracy of better than $1 \%$ of a pixel. Because HST images are undersampled even by the PC1, we have designed a program of 15 exposures per epoch that incorporates a dithering pattern of sub-pixel shifts with different values in order to improve the spatial resolution. This enables us to regain the oversampling of the $\mathrm{PCl}$ images required for high-precision astrometry.

- The last requirement is to have a distant reference frame. Fortunately, this is a simple problem to solve in the case of 47 Tuc. A background of stars in the SMC provides a rich reference frame of stars which can be easily identified on color-magnitude diagrams (see 


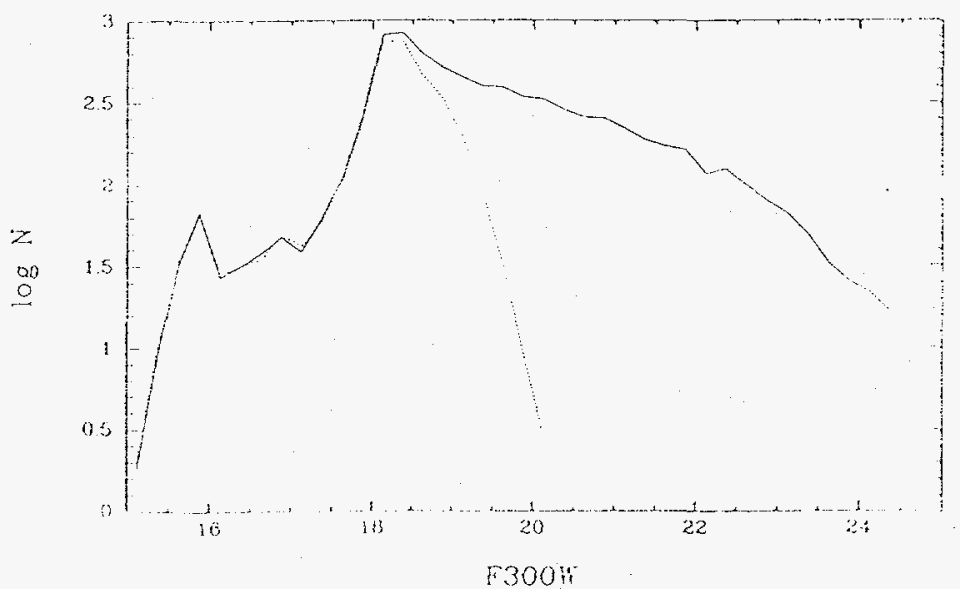

Figure 1. Luminosity function of the 47 Tuc core (solid line). No comecion has been applied for incompleteness. Note the HB peat at F300W $\approx 16$ and the TO peak at $\mathrm{F} 300 \mathrm{~W} \approx 18$. The LF of stars with F555W photometry from Guhathaturta et al. (1992) is shown for comparison (dotted line).

Hesser et al. 1987). These numerous $F$-type stars should appear at $U=21.0-220$ mag Unfortunately, the photometry published by Guhathakurta et al. (1992) is not deep emongh to allow us to currently identify these SMC stars (or any WDs in 47 Tuc).

Based on tests with WFPC1 frames, a realistic goal for the precision of our final proper motions is a $1-\sigma$ limit of 0.23 mas/yr, which would allow a $5-\sigma$ detection of all stars with tangential velocities $\geq 22 \mathrm{~km} \mathrm{~s}^{-1}$ in the WFPC2 frames (PC field).

\section{Astrophysical By-products:}

The first-epoch observations of our project already provide a number of very interming astrophysical by-products, namely:

- deep luminosity functions in the UV:

- deep color-magnitude diagrams (CMDs):

- variable stars (pulsating stars and binaries); and

- the identification of possible UV counterparts for known X-ray sources (these LMARBS or $C V$ 's being accreting binary systems with neutron star or white dwarf primarise mur tively).

\subsection{Luminosity Functions}

Figure 1 shows the F30017 (instrmentall luminosity function for the stars in the l'e thed. The brighter peat at 15.8 mag corresponds 10 the RHB stars and the fanter peati di 1 .5 mag to the MS turnoff (TO). Note that there are no saturated stass in any of on 350 ser F300W images; therefore, they are complete for $\mathrm{F} 300 \mathrm{~W} \leq 20 \mathrm{mag}$.

\subsection{Color-Magnitude Diagrams.}

Figure 2 presents two color-magnitude diagrams for the core of 47 Tuc (PC1 data only). All stages of stellar evolution in the cluster are present in our data: TO stars with $\mathcal{M} \sim 0 . \mathcal{M}$. lower $M S$ stars with $M \geq 0.5 M_{\odot}$. RGB stars, HB stars, AGB stars, BS stars, and WD stars. (Though WDs must be present in our data, we are not able to show them in Fig. 2 because the complementary optical photometry is not deep enough yet.) 

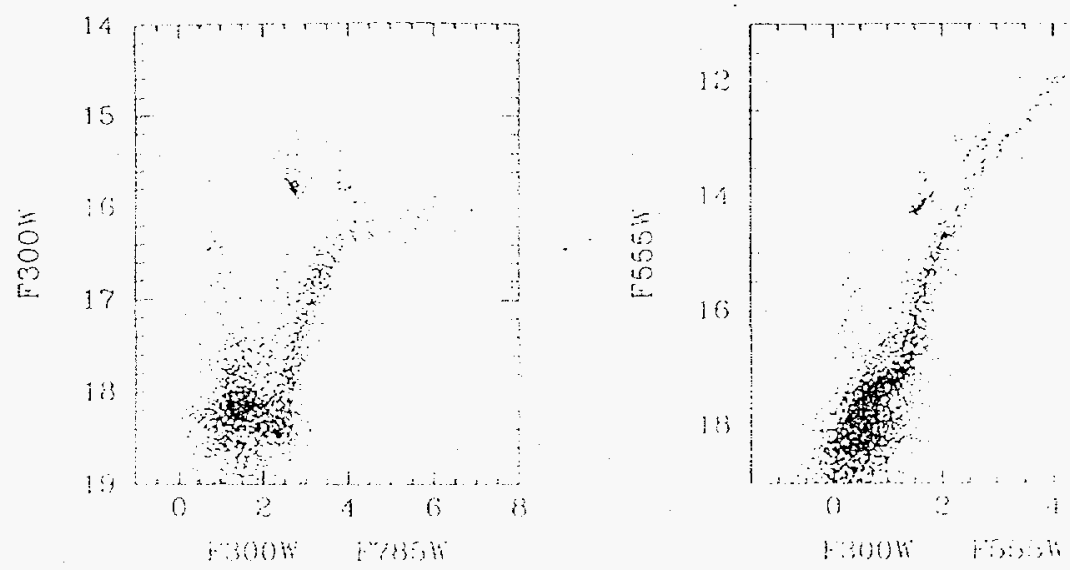

Figure 2. Color-magnitude diagrans using our F300W observations along with P555W and F785x data from Guhathakura et al. (1992).

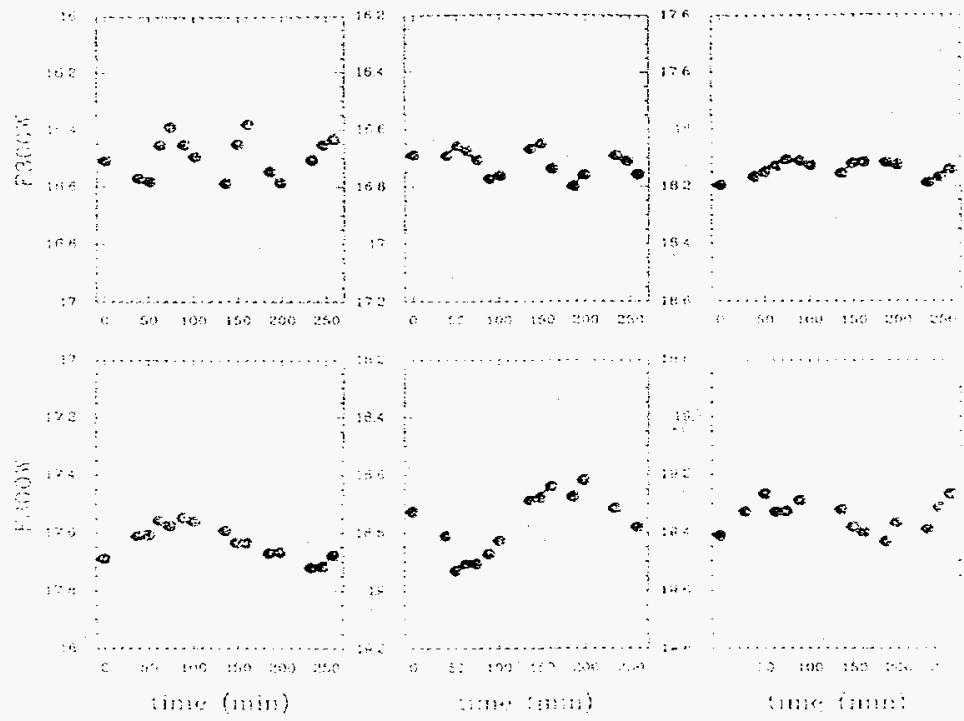

Figure 3. Examples of light curves for 6 variable blue stragglers. Lipper panels: pulsating variables of SX Phe type. Lower panels: semi-detached eclipsing binaries, for which Gilliland et al. (1995) and Edmonds et al. (1996) show the entire moriod.

\subsection{Variability of Blue Stragglers}

Since there are no saturated stars and our photometry is complete for $13001 \mathrm{~S} 20$ mat. it is possible to obtain a complete sample of BS. RHB, and RGB stars in the t7 Tuc core. All stars brighter than F300W $=20 \mathrm{mag}$ have P555W photometry from Guhathakurta al. (1992).

Note that we are able to identify more blue stragglers (a total of 34 ) than any previous work because of (i) the refurbished HST, (ii) the brightness of these stars in the UV, and (iii) our faint limit of completeness. For comparison, Paresce et al. (1991) and De Marchi et al. (1993) found 21 blue stragglers and Guhathakurta et al. (1992) found 11 blue stragglers.

The time coverage and resolution of our observations ( 15 frames within about 3 hours) makes these data ideal for the detection of short-period variables with small amplitudes $(\geq 0.05 \mathrm{mag}$ for $\mathrm{F} 300 \mathrm{~W}<17 \mathrm{mag}$ and $\geq 0.20 \mathrm{mag}$ for $\mathrm{F} 300 \mathrm{~W}<20 \mathrm{mag}$, for example). Variables in the period range $1^{h} \leq P \leq 8^{h}$ are semi-detached binaries or pulsating stars of SX Phe type (e.g., Nemec ot a. 1994). 


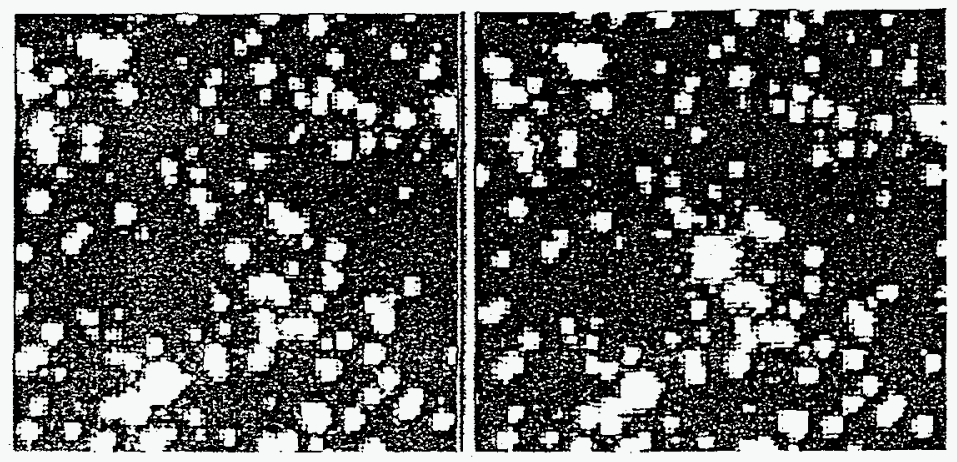

Higure 4. Field of the variable star AKO 9, which increased by more than 2 magnitudes in less than 1 hour (left: our first frame, right: our last frame). The field size is $3.9 \times 3.9$ arcsec, centered on AKO 9 .

A total of 25 photometrically variable stars have been identified in the PC1 frame

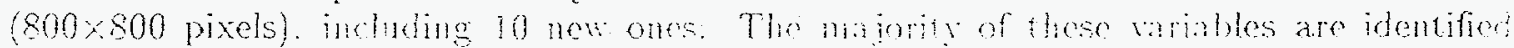
with blue straggler or blue straggler candidate stars. The variables observed by Gilliland et al. (1995) and Edmonds et al. (1996) are present (11 out of 13), as well as the variables $\checkmark 1$ of Paresce et al. (1992) and V2 of Paresce C De Marchi (1994). Tigure 3 displays six of our light-curves for variable blue stragglers.

It is worth mentioning that our data are nicely complementary to the data of Gilliland et al. (1995): the time coverage of their WFPC1 observations favor the observations of variables with $P \geq 0.5$ daz, while our WFPC2 data favor variables with $P \leq 0.5$ day.

We have detected $7 \mathrm{SX}$ Phe pulsating variables, wh periods ranging from 1.2 to $1 . \mathrm{s}$ hours. Two of them are bhe siraggess and have ben provously identifed by Edmonds al. (1996). The rest of them are blue straggler candidates, in the sense that they are bluer and brighter than the main sequence of the clisor, but within the photometric scatter of this sequence, as shown in Figure 2 .

We have found 9 candidates for short-period binary stars in the PCl frame alone. including 4 previously identified by Edmonds a al (1996). The longer-period binaries observed by Edmonds et al. (1996) were also examined and we confirm their variability. although we do not have sufficient time coverage to confirm their periods. The variables $V 1$ and $V 2$ of Paresce o! al. (1992, 1994) were quiescen! in our data: their olserved magnitude ranges are consistent wh the photometric enrors.

\subsection{The puzzling variable AKO 9}

Buring the rather short total time inerval of all onr observations (about 3 lours) one sta in the core of 47 Tuc had the good will to morease in luminosicy by more han 2 magnitudein less than one hour. By the end of our observations this star had become the brightest UV source in the core. We have identified it with shar 9 of Auriere et al. (1989) and stai \# 2059 of De Marchi et al. (1993). Figure 4 shows the field of this variable star, named AKO 9 following the first paper mentioning it. The left panel shows our first observation and the right one our last. Figure 5 displays our light curve for AKO 9 during the whole time interval of our observations. AKO 9 is an eclipsing binary with a period of 1.1 day and an amplitude of $1.2 \mathrm{mag}$ (Gilliland et al. 1995). At the beginning of our observations. AKO 9 was at its usual magnitude level (as a variable star), but it subsequently increased in brightness by more than 3 times the variation due to its eclipses.

Only Aurière et al. (1989) has observed AKO 9 during a flare similar to ours. This star was identified as the possible UV counterpart within the error box of the EINSTEIN variable 


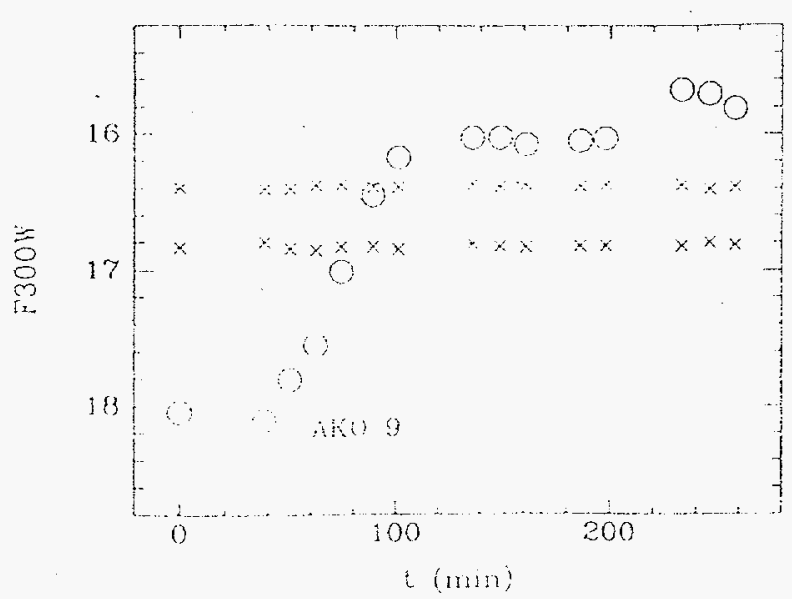

Figure 5. Light curves for AKO 9 and two stars of constant brightness.

X-ray source X0021.8-7221 (Aurière et al. 1993). ROSAI observations show multiple X-ray

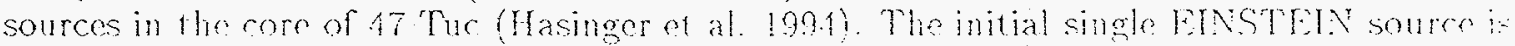
resolved into five components - X5, X7, X8, X9 and X10 of which X8, X9 and XJ0 a themselves variable, according to Hasinger al al. (1993). If we identify AKO 9 with the $X 9$ source, then each other $X$-ray source lies within 1 arcsec of the position of a blue straggler (except for $\mathrm{X} .5$. which lies close to the border of our PCl frame). This is a very significant result, which may support the idea that these individual $X$-ray sources are too bright to be CVs, as argued by Verbunt (1996). Note that there is precedent for blue stragglers being $X$-ray sources with $L_{X} \sim 10^{3.3} \mathrm{erg} \mathrm{s}^{-1}$ in the open cluster $\mathrm{M} 6 \mathrm{i}$.

\section{Conclusions}

As.uming that we are able to obtain HST/WFPC2 observations with the same roll angk and with the same positions to within a few pixels, we will easily obtain positions accumate to $1 / 100^{\text {th }}$ of a pixel in the globular cluster $t i$ Tucanae and thus, $1-\sigma$ proper motion measurenents of 0.23 mas/y over the course of 3 crcles, i.e, about two years. Consequentr. HST can measure accurate proper motions in the cores of globular clusters with a reasonably short time baseline. This absolutely unique facility should be used, perhaps by devoting some fraction of HST time to the acquisition of $2^{\text {nd }}$ epoch frames for faint sources in olluer globular clustens, in the Galactic halo and bulge. and in nearby dwarf spheroidal galaxics.

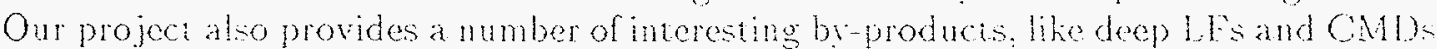
(Figures 1 and 2) and information on short-tinescale varability in the ore of 47 Tuc: (Figures 3-5). For the first tme, we have detemined that we luminous ROSAT X-ra! sources XT, Xs, and X10 in the core of 47 Tuc are asociated with blue straggler stars. Also, the source X9, associated with the echipsing binary star hliO 9. experienced, during our 3 hours of observations, an increase in luminosity of more than 2 magnitudes in less than one hour. This binary star is indeed very interesting: perhaps on its way to also becoming a blue straggler.

Acknowledgments. We are very grateful to R. Guhathakurta for sending us his photometry files, and to J. Liebert and $\mathrm{F}$. Verbunt for useful discussions and comments.

U. S. Department of Energy by Lawrence Livermore National Laboratory under Contract W-7405-Eng-48. 


\section{References}

Antonov V.A., 1962, in IAU Symp. 113, p. 525

Aurière M., Koch-Miramond L., Ortolani S. 1989, A\&, 214, 113

Aurière M., Laurezal, C., Koch-Miramond E. 1993, in "Frontiers of Space and Ground Based Astronomy", eds. W. Wamsteker et al. (Springer), p. 633

De Marchi G., Paresce, F., \& Ferraro, F. R. 1993, Ap.JS, 85, 2933

Djorgotski S., Piotto G., 1993, ASP Conf. Series Vol: 50, p. 2013

Edmonds P.D., Gilliand R.L., Guhathakuta P., Petro 1.1), Saha A., Shara M.M. 1996, Ap.T, submitted

labian A., Pringle J., Rees M., 1975, MNRAS, 172, 15l

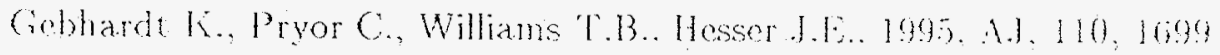

Gilliland R.L., Edmonds P.D., Petro L., Saha A., Shara M.M., 1995, Ap.l, 147, 191

Guhathakurta P., Yanny B., Schneider D.P., Bahcall .J.N. 1992, A.J. 101, 1790

Hasinger G., Johnston H.M., Verbunt F. 1994, ACA, 28s. 160;

Hergie D.C. 1975, MNRAS. 173. 729

Héron M., 1961, Ann d'Astrophys, 24, 369

Hesser J.E., et al. 1987, PASP, 99. 739

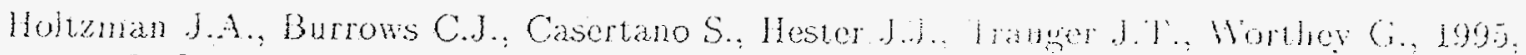
PASP, 107, 156

Hut P., et al., 1992, PASP, 104, 981

Lynden-Bell D. Wood R., 1968, MNRAS, 138, 495

Mancholer R.N. et al., 1991, Nat. 352, 219

Niman G. 1983, A\&A, 214. 106

Meylan G., 1994, in Lecture Notes in Physics, Vol. 430. 1). 22

Meytali (i., et al., 1991, Ap J, 383, 587

None D.C., et al. 1992, A.J, 103.638

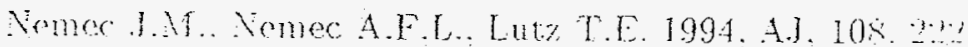

Paresce F., et al., 1991, Na.t. 352, 297

Paresce F. De Marchi G., 1994, ApJ, 427, L33

Paresce F., De Marchi G., Ferraro F.R. 1992. Nat. 360 . th

Phimney E.S. Sigurdsson S.. 1991, Nat, 349, 220

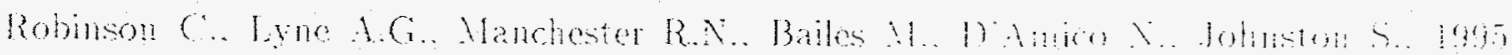
MNRAS. 27A, J4

Sigudsson S. Phimey E.S. 1995 . Ap.IS. 99.609

Spitzer $1 ., 1969, A, J, 158,1,139$

Timey C.C., 1993, A., 105, 1169

Verbunt F, 1996, in IAU Symp. 17 "Dynamical Exolution of Star Clusters". eds. I. Matino \& P. Ifut (Kluwer: Dordrecht), in press 